\title{
QT dispersion and left ventricular morphology in patients with hypertrophic cardiomyopathy
}

\author{
K Sakata, M Shimizu, H Ino, M Yamaguchi, H Terai, K Hayashi, M Kiyama, T Hayashi, \\ M Inoue, H Mabuchi
}

See end of article for authors' affiliations

Correspondence to: Dr Masami Shimizu, Molecular Genetics of Cardiovascular Disorders, Division of Cardiovascular medicine, Graduate School of Medical Science,

Kanazawa University

Takara-machi 13-1

Kanazawa 920-8640,

Japan; shimizu@

med.kanazawa-u.ac.jp

Accepted

19 February 2003

\begin{abstract}
Objective: To evaluate the relation between QT variables and disproportion of left ventricular wall hypertrophy in patients with hypertrophic cardiomyopathy.

Design: Retrospective analysis of the results of echocardiography and electrocardiography.

Setting: University hospital (tertiary referral centre).

Patients: 70 patients with hypertrophic cardiomyopathy were divided into four groups according to the distribution of left ventricular wall hypertrophy on cross sectional echocardiography: group A-hypertrophy confined to the interventricular septum; group B-hypertrophy confined to the interventricular septum and left ventricular anterior wall; group C-hypertrophy confined to the interventricular septum, left ventricular anterior wall, and lateral free wall; group D-hypertrophy involving all portions of the left ventricle.

Main outcome measures: QT intervals and QT dispersion in precordial six lead ECGs.

Results: There were no significant differences in the maximum left ventricular wall thickness among the four groups, and maximum and minimum QTc intervals also did not differ. QTc dispersion was increased significantly in groups $A$ and $B$ compared with groups $C$ and $D$. Dispersions of the interval from the J point to the end of the T wave (JTc dispersions) in groups $A$ and $B$ were also increased significantly compared with groups $C$ and D. By linear regression analysis, QTc and JTc dispersions correlated with the ratio of the interventricular septal thickness to left ventricular posterior wall thickness ( $p=0.0152$ and $p=0.0075$, respectively).

Conclusions: QT dispersion may be affected by not only electrical inhomogeneity but also by morphological inhomogeneity of the left ventricle in patients with hypertrophic cardiomyopathy.
\end{abstract}

E xperimental studies have suggested that variability between the ECG leads in measurement of QT intervals and QT dispersion reflects spatial and temporal inhomogeneity of left ventricular recovery times. ${ }^{1-3}$ Previous clinical studies of QT dispersion have shown that it is increased in patients with hypertrophic cardiomyopathy, ${ }^{45}$ and also in patients with the long QT syndrome ${ }^{6}$ and those with myocardial infarction. ${ }^{17}$ In patients with hypertrophic cardiomyopathy, prolonged QT dispersion has been related to an increased risk of serious ventricular arrhythmias or sudden death..$^{8-10}$ In contrast, other investigators found no significant association between QT dispersion and any risk factor for sudden cardiac death in patients with hypertrophic cardiomyopathy, ${ }^{11}$ and concluded that QT dispersion was not a reliable predictor of sudden death in this disorder. ${ }^{12}$ Assessment of QT dispersion for risk stratification should be interpreted carefully. Therefore, evaluation of QT dispersion might provide clinical characterisation of patients with hypertrophic cardiomyopathy, but its relation to sudden cardiac death remains uncertain.

Most patients with hypertrophic cardiomyopathy present with asymmetrical hypertrophy of the septum and anterior wall of the left ventricle, and disproportionate wall hypertrophy is characteristic in this disease. Wall hypertrophy increases activation and recovery time, and prolongs QT interval. Accordingly, it is hypothesised that wall hypertrophy or inhomogeneity of left ventricular wall thickness in patients with hypertrophic cardiomyopathy may affect QT variables, especially QT dispersion - that is, morphological inhomogeneity may lead to electrophysiological inhomogeneity in patients with this disease. However, this hypothesis has not been proven to date. Our aim in the present study was to assess whether inhomogeneity of the distribution of left ventricular wall hypertrophy affects QT variables in patients with hypertrophic cardiomyopathy.

\section{METHODS}

\section{Study subjects}

We evaluated 70 patients with hypertrophic cardiomyopathy (15 female and 55 male; age range 19-81 years, mean 56 years). The diagnosis was based on the echocardiographic demonstration of a non-dilated hypertrophied left ventricle in the absence of other cardiac or systemic causes for left ventricular hypertrophy. ${ }^{13}$ Fifty six patients (80\%) with hypertrophic cardiomyopathy had an asymmetrical hypertrophy, with a ratio of septal to posterior wall thickness of 1.3 or greater. Patients with atrial fibrillation, conduction disturbances, or an inadequate ECG for evaluating QT variables were excluded from the study. Patients with apical hypertrophy or with dilated cardiomyopathy were also excluded.

We defined wall hypertrophy as a wall thickness of more than $13 \mathrm{~mm}$. The subjects were divided into four groups according to the distribution of left ventricular wall hypertrophy on cross sectional echocardiography: group A included patients with hypertrophy confined to the septum; group B included patients with hypertrophy confined to the septum and left ventricular anterior wall; group $\mathrm{C}$ included patients with hypertrophy confined to the septum, left ventricular

Abbreviations: IVST, interventricular septal thickness; JT, interval from the J point to the end of the T wave; PWT, left ventricular posterior wall thickness; QTp, interval from the onset of the QRS complex to the peak of the $T$ wave; Tp, the interval from the peak of the $T$ wave to its end 


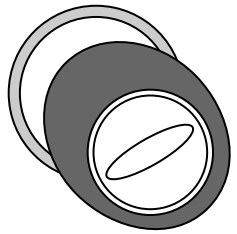

A

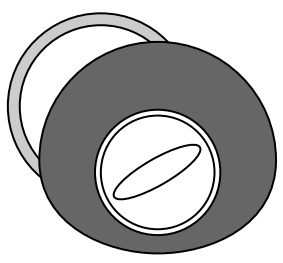

C

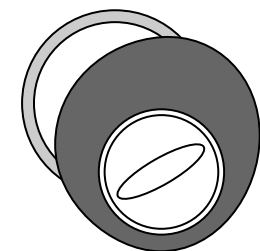

B

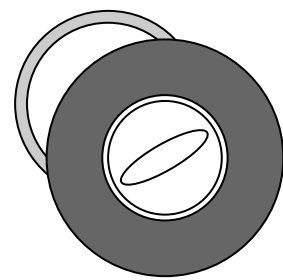

D
Figure 1 Schema of morphological patterns of left ventricular wall hypertrophy at the level of the tips of the mitral valve leaflets from the left ventricular short axis view on cross sectional echocardiography. (A) Hypertrophy confined to the septum. (B) Hypertrophy confined to the septum and left ventricular anterior wall. (C) Hypertrophy confined to the septum, left ventricular anterior wall, and lateral wall. (D) Hypertrophy of all portions of left ventricle.

anterior wall, and lateral wall; and group D consisted of patients with hypertrophy of all portions of the left ventricle (fig l).

\section{Measurement of QT interval and dispersion}

All ECGs were recorded at $25 \mathrm{~mm} / \mathrm{s}$ with standard lead positions, and all records were magnified by $200 \%$ to improve resolution. QT intervals, the onset of the QRS complex to the peak of the T wave (QTp) intervals, and QRS duration were measured in each lead of the precordial six lead ECGs (Vl-V6). QT intervals were measured from the onset of the QRS complex to the end of the T wave. The end of the T wave was defined as the intersecting point of a tangent line on the terminal $\mathrm{T}$ wave and the TP baseline. When $\mathrm{U}$ waves were present, the QT interval was measured to the nadir of the curve between the $\mathrm{T}$ and $\mathrm{U}$ waves. The QRS complex duration was measured from the beginning of the QRS complex to its end. The interval from the peak of the $\mathrm{T}$ wave to its end (Tp) was calculated from the equation $\mathrm{Tp}=\mathrm{QT}-\mathrm{QTp}$, and the JT interval (from the J point to the end of the T wave) was calculated from the equation JT $=\mathrm{QT}-\mathrm{QRS}$. The dispersions of QT, QTp, JT, Tp, and QRS were calculated as the differences between the maximum and minimum QT, QTp, JT, Tp, and QRS values, respectively. Values of the QT, QTp, JT, and Tp intervals and dispersions were corrected for heart rate using Bazett's formula (QTc, QTpc, JTc, and Tpc, respectively). If the height or depth of the T wave was $<1.5 \mathrm{~mm}$, its lead was excluded from analysis.

In order to eliminate both interobserver variability and bias, QT intervals were measured using a digitiser in each of the six precordial leads by a single observer who was blinded to all clinical and echocardiographic findings. The intraobserver measurement variability was calculated by repeated measurements of the same ECGs by the same observer. The mean of absolute values of intraobserver variability of QTc dispersion for this observer was $3 \mathrm{~ms}$, and mean difference relative to mean value of QTc dispersion was 6\%.

\section{Echocardiographic examinations}

Standard transthoracic M mode and cross sectional echocardiographic studies were done to identify and quantify morphological features of the left ventricle, using criteria established by the American Society of Echocardiology. ${ }^{14}$ The thicknesses of the interventricular septum (IVST) and left ventricular posterior wall (PWT) were measured at the level of the tips of the mitral valve leaflets, and the IVST/PWT ratio was calculated. Fractional shortening was calculated as the difference in end diastolic and end systolic dimensions divided by the end diastolic dimension. The parasternal short axis view was used to assess the degree and disproportion of left ventricular wall hypertrophy. At the level of the tips of the

\begin{tabular}{|c|c|c|c|c|}
\hline & Group A & Group B & Group C & Group D \\
\hline Number of cases & 9 & 21 & 26 & 14 \\
\hline Male & $7(78 \%)$ & $16(76 \%)$ & $20(77 \%)$ & 12 (86\%) \\
\hline Age (years) & $56(18)$ & $54(15)$ & $56(14)$ & $61(9)$ \\
\hline History of syncope & $1(11 \%)$ & $3(15 \%)$ & $4(15 \%)$ & $2(14 \%)$ \\
\hline Family history of SCD & $1(11 \%)$ & $6(30 \%)$ & $6(22 \%)$ & $7(50 \%)$ \\
\hline VT & $2(22 \%)$ & $3(14 \%)$ & $3(13 \%)$ & $1(7 \%)$ \\
\hline \multicolumn{5}{|l|}{ Echocardiogram } \\
\hline $\operatorname{maxWT}(\mathrm{mm})$ & $17.7(4.4)$ & $19.7(4.0)$ & $20.0(4.9)$ & $17.9(5.1)$ \\
\hline IVST (mm) & $16.6(3.2)$ & $18.2(3.6)$ & $18.9(4.6)$ & $17.6(5.2)$ \\
\hline PWT (mm) & $11.0(1.5)^{*}$ & $12.0(1.4)^{*}$ & $12.1(2.1)^{*}$ & $14.7(1.4)$ \\
\hline IVST/PWT & $1.55(0.48)$ & $1.55(0.34) \dagger$ & $1.57(0.30) \dagger$ & $1.19(0.28)$ \\
\hline $\operatorname{LAD}(\mathrm{mm})$ & 39.7 (4.5) & $40.0(7.9)$ & $39.6(6.4)$ & $39.6(6.8)$ \\
\hline LVDd (mm) & 46.7 (7.3) & $44.2(7.2)$ & $45.1(5.5)$ & $44.3(5.3)$ \\
\hline LVDs (mm) & $30.0(5.2)$ & $26.2(7.2)$ & $27.5(4.4)$ & $26.8(3.8)$ \\
\hline FS $(\%)$ & $35.7(5.3)$ & $41.1(9.9)$ & $39.0(6.4)$ & $42.0(9.6)$ \\
\hline \multicolumn{5}{|l|}{ Drug treatment } \\
\hline$\beta$ Blocker & $2(22 \%)$ & $3(15 \%)$ & $8(30 \%)$ & $2(14 \%)$ \\
\hline Calcium antagonist & $4(44 \%)$ & $8(40 \%)$ & $12(44 \%)$ & $2(14 \%)$ \\
\hline ACEI or ARB & $1(11 \%)$ & $3(14 \%)$ & $2(8 \%)$ & $4(29 \%)$ \\
\hline Amiodarone or sotalol & 0 & 0 & $1(4 \%)$ & 0 \\
\hline \multicolumn{5}{|c|}{$\begin{array}{l}\text { Values are mean (SD) unless stated. } \\
\text { Group A: patients with hypertrophy confined to the septum; group B: patients with hypertrophy confined to } \\
\text { the septum and left ventricular anterior wall; group C: patients with hypertrophy confined to the septum, left } \\
\text { ventricular anterior wall, and lateral wall; group D: patients with hypertrophy of all portions of the left } \\
\text { ventricle. } \\
\text { *p< } 0.001 \text { v group D; †p } 0.05 \text { v group D. } \\
\text { ACEI, angiotensin converting enzyme inhibitor; ARB, angiotensin II receptor blocker; FS, fractional } \\
\text { shortening; IVST, interventricular septal thickness; LAD, left atrial dimension; LVDd, left ventricular end } \\
\text { diastolic dimension; LVDs, left ventricular end systolic dimension; maxWT, maximum LV wall thickness; PWT, } \\
\text { left ventricular posterior wall thickness; SCD, sudden cardiac death; VT, ventricular tachyarrhythmia. }\end{array}$} \\
\hline
\end{tabular}




\begin{tabular}{|c|c|c|c|c|}
\hline & Group A & Group B & Group C & Group D \\
\hline RR interval (ms) & 907 (185) & $969(190)$ & $960(146)$ & 970 (132) \\
\hline \multicolumn{5}{|c|}{ QTc interval (ms) } \\
\hline Max & $449(22)$ & $447(31)$ & $440(20)$ & $426(26)$ \\
\hline Min & $375(16)$ & $382(35)$ & $402(24)$ & $395(27)$ \\
\hline \multicolumn{5}{|c|}{ QTpc interval (ms) } \\
\hline Max & $348(28)$ & $354(18)$ & $357(21)$ & $352(28)$ \\
\hline Min & 297 (9) & 297 (32) & $308(27)$ & $305(26)$ \\
\hline \multicolumn{5}{|c|}{ QRS duration (ms) } \\
\hline Max & 105 (12) & $100(13)$ & $97(10)$ & 97 (7) \\
\hline Min & $84(9)$ & 85 (11) & $82(10)$ & $83(5)$ \\
\hline \multicolumn{5}{|c|}{ Tpc interval (ms) } \\
\hline Max & $128(30)$ & $128(32)$ & $112(29)$ & $113(22)$ \\
\hline Min & $52(12)$ & $60(17)$ & $65(16)$ & 55 (14) \\
\hline \multicolumn{5}{|c|}{ JTc interval (ms) } \\
\hline Max & $353(27)$ & $353(29)$ & $350(23)$ & $336(28)$ \\
\hline Min & 274 (23) & $286(33)$ & $311(26)$ & $300(32)$ \\
\hline \multicolumn{5}{|c|}{ Dispersion (ms) } \\
\hline Qtc & $74(24)^{*} \dagger$ & $65(28)^{*} \dagger$ & $39(17)$ & $31(15)$ \\
\hline Qtpc & $51(27)$ & $57(27)$ & $48(21)$ & $46(23)$ \\
\hline QRS & $21(11)$ & $15(9)$ & $15(9)$ & $14(7)$ \\
\hline Tpc & 77 (32) & 68 (33) & $48(28)$ & $58(20)$ \\
\hline JTc & $79(37)^{\star} \ddagger$ & $67(26)^{*} \ddagger$ & $40(18)$ & $36(19)$ \\
\hline \multicolumn{5}{|c|}{ 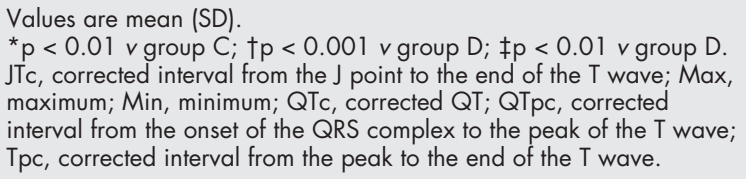 } \\
\hline
\end{tabular}

mitral valve leaflets the ventricle was divided into four regions that identified the ventricular septum and the anterior, lateral, and inferior left ventricular wall (fig l). The hypertrophied region $(>13 \mathrm{~mm}$ ) was estimated from these four different regions of the left ventricle at end diastole.

\section{Statistical analysis}

Values are expressed as the mean (SD). Comparison between the groups was done using a one way analysis of variance (ANOVA) followed by Scheffé's method. Categorical data were compared using $\chi^{2}$ analysis. Correlation was assessed by linear regression analysis and Pearson's correlation coefficient. A probability value of $\mathrm{p}<0.05$ was considered significant. StatView 5.0 (Abacus Concepts Inc, Berkeley, California, USA) was used for data analysis.

\section{RESULTS}

\section{Clinical and echocardiographic data}

Group A consisted of nine patients ( $12.9 \%$ ), group B consisted of 21 patients $(30.0 \%)$, group $C$ consisted of 26 patients $(37.1 \%)$, and group D consisted of 14 patients $(20.0 \%)$. Clinical

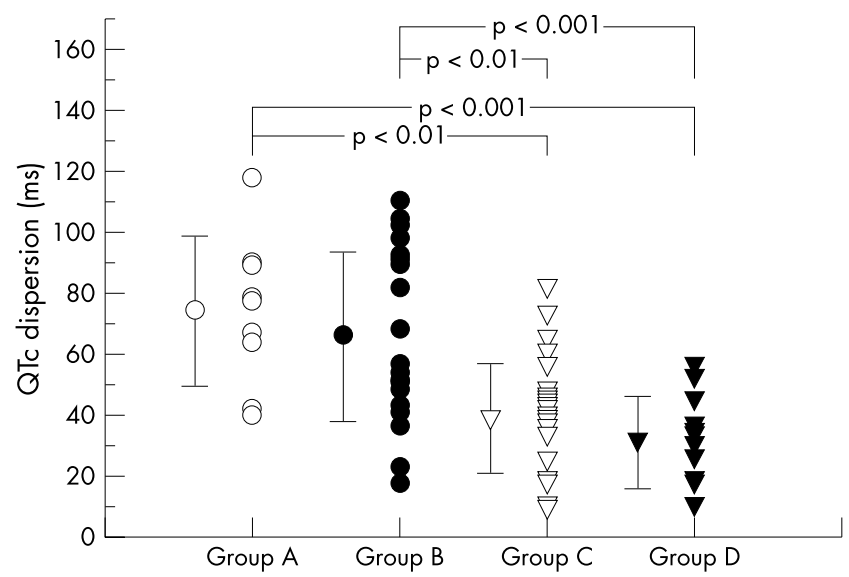

Figure 2 QTc dispersion in the four groups. Vertical bars represent SD.
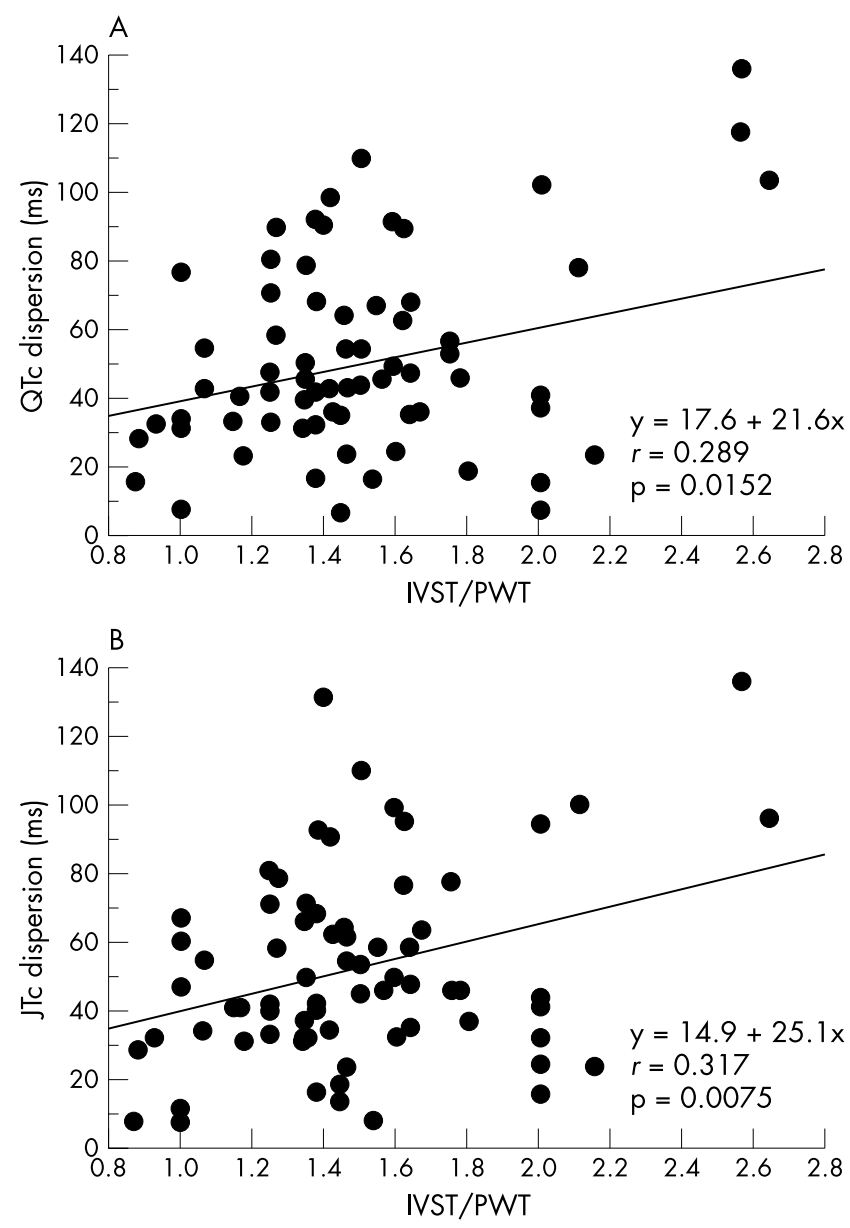

Figure 3 Relation between QTc dispersion and IVST/PWT (panel A), and between JTc dispersion and IVST/PWT (panel B). IVST, interventricular septal thickness; PWT, left ventricular posterior wall thickness.

characteristics, echocardiographic data, and drug treatment of the study subjects are summarised in table l. There were no significant differences in the clinical characteristics or drug treatment among the four groups. On echocardiography there were no significant differences in the maximum left ventricular wall thickness and the IVST among the four groups. PWT was significantly greater in group D than in the other groups, and IVST/PWT was significantly smaller in group D than in groups $\mathrm{B}$ and $\mathrm{C}$.

\section{QT variables}

The results of QT interval analysis are summarised in table 2. The maximum and minimum QTc did not differ among the four groups. The QTpc, QRS, Tpc, and JTc intervals also did not differ significantly, and average heart rate was similar among the four groups as well. In contrast, QTc and JTc dispersions were greater in both groups A and B than in groups C and D (fig 2). By linear regression analysis, QTc and JTc dispersion did not correlate with maximum wall thickness $(r=0.071$, $\mathrm{p}=0.559$, and $r=0.029, \mathrm{p}=0.8096$, respectively). However, there was a significant correlation between IVST/PWT and both QTc dispersion $(r=0.289, \mathrm{p}=0.0152)$ and JTc dispersion $(r=0.317, \mathrm{p}=0.0075)$, as shown in fig 3 .

\section{DISCUSSION}

In this study we show that QTc dispersion was more prolonged in patients with hypertrophic cardiomyopathy in whom hypertrophy of the left ventricular wall was confined to the interventricular septum and anterior wall (groups A and B) than in those with more extensive left ventricular hypertrophy (groups C and D). The results suggest that increased QT 
dispersion on the surface ECG may be affected by not only electrical inhomogeneity (ventricular repolarisation) but also by morphological inhomogeneity of the left ventricle.

Several studies have suggested that maximum QT interval and QT dispersion are prolonged in patients with hypertrophic cardiomyopathy. ${ }^{4} 8$ 10-12 An increase in QT dispersion represents regional differences in myocardial repolarisation, and this phenomenon may be associated with an increased risk of malignant ventricular tachyarrhythmias and sudden death, especially in patients with hypertrophic cardiomyopathy. ${ }^{8-10}$ Yetman and colleagues reported ${ }^{10}$ that greater QT dispersion on the ECG was strongly associated with an increased risk of mortality. In contrast, whether increased QT dispersion may contribute to the induction of electrical instability-which in turn may lead to malignant ventricular tachyarrhythmias and sudden cardiac death in some individuals with hypertrophic cardiomyopathy-remains unclear. ${ }^{11}{ }^{12}$ In the present study QTc dispersion was more prolonged in groups A and B than in groups $C$ and $D$, although the history of syncope and ventricular tachycardia did not differ among the four groups. Moreover, there was no relation between increased QT dispersion and ventricular tachycardia in our patients (data not shown). These results suggest that the assessment of QT dispersion as a risk stratification in patients with hypertrophic cardiomyopathy is controversial. On the other hand, this disease is characterised by left ventricular hypertrophy that is usually asymmetrical, affecting various regions of the ventricle.

Because wall hypertrophy can modify both activation and recovery time, we hypothesised that wall hypertrophy or disproportion of left ventricular wall thickness in patients with hypertrophic cardiomyopathy could affect QT dispersion. Although maximum wall thickness did not differ among the four groups in our study, QTc dispersion was significantly greater in groups $A$ and $B$ than in groups $C$ and $D$, providing some credence for our hypothesis. Moreover, QTc dispersion showed a positive correlation with IVST/PWT. These results suggest that in addition to repolarisation instability, disproportion of left ventricular hypertrophy may be one of the factors that affects QT dispersion on the surface ECG in patients with hypertrophic cardiomyopathy. An increased QT dispersion has been also reported in hypertensive patients with left ventricular hypertrophy. ${ }^{15-17}$ Zaidi and colleagues have reported $^{5}$ that the QT dispersion and the maximum QT interval were significantly longer in patients with hypertrophic cardiomyopathy than in patients with left ventricular hypertrophy of other origin. Symmetrical left ventricular hypertrophy is more common among subjects with arterial hypertension than in those with hypertrophic cardiomyopathy. These reports also support our hypothesis. In contrast, QT dispersion did not correlate with maximum wall thickness in our study. Dritsas and colleagues also reported ${ }^{4}$ that QT dispersion was not associated with maximum wall thickness in patients with hypertrophic cardiomyopathy. These results suggest that the magnitude of left ventricular wall hypertrophy itself may not be a major factor affecting QT dispersion. In our study both QTc and JTc dispersion were greater in groups $\mathrm{A}$ and $\mathrm{B}$ than in groups $\mathrm{C}$ and $\mathrm{D}$, and QRS duration did not differ among the four groups. From these results, it is suggested that inhomogeneity of the distribution of left ventricular wall hypertrophy may play a role in increasing QT dispersion in patients with hypertrophic cardiomyopathy, and that this increase in QT dispersion may be caused by the inhomogeneity of regional repolarisation.

Although the aetiology of increased QTc dispersion has not been well defined in patients with hypertrophic cardiomyopathy, myocardial disarray and interstitial fibrosis may cause the inhomogeneity of ventricular refractoriness and intraventricular conduction, and may increase QTc dispersion. ${ }^{11}$ Moreover, our study showed that the QTc dispersion in hypertrophic cardiomyopathy was influenced by the disproportionate left ventricular hypertrophy. From these findings, QTc dispersion in this disorder may be affected by macroscopic spatial inhomogeneity related to morphological patterns of the left ventricle in addition to microscopic electrical inhomogeneity in the myocardium. Additionally, the association between QT dispersion and risk factors for sudden cardiac death in patients with hypertrophic cardiomyopathy is varied in previous studies, ${ }^{8-11}$ because QT dispersion in patients with hypertrophic cardiomyopathy may be influenced by various factors such as inhomogeneity of distribution of left ventricular wall hypertrophy. Quantification of the risk of sudden death remains imprecise for most patients with hypertrophic cardiomyopathy. Several risk factors have been identified, but current stratification profiles are inadequate to identify all patients who are at high risk for sudden death. On the other hand, Spirito and colleagues have reported that the magnitude of hypertrophy is directly related to the risk of sudden death and is a strong and independent predictor of prognosis. ${ }^{18}$ Our study showed that increased QT dispersion, believed by some to be a marker of the risk of sudden death, may be influenced by the disproportionate left ventricular hypertrophy. Therefore it is possible that patients with more asymmetrical forms of hypertrophy may be at high risk of sudden death. Of our 70 patients, only two who were categorised in group B died suddenly from causes related to their hypertrophic cardiomyopathy. Because the sample size is too small, it is difficult to assess the prognostic data for sudden death in this disease according to the distribution of left ventricular hypertrophy. Additional studies including larger samples are necessary to confirm and clarify our results.

In this study we measured the QT intervals in each lead of the precordial six lead ECG (Vl-V6). The rationale for this was based on the fact that the unipolar precordial leads presumably reflect local ventricular activity to some degree, ${ }^{19}$ and may therefore individually reflect local variations in repolarisation. The bipolar limb leads, on the other hand, may, according to Einthoven's equation, be incapable of reflecting such heterogeneity. Thus QT dispersion measured by recording leads VI-V6 probably provides a better index than that measured from a full 12 lead ECG. Further investigations are necessary in future to settle this point.

\section{Study limitations}

We divided the subjects into four different types of left ventricular morphology from the left ventricular short axis view on cross sectional echocardiography. Consequently, one limitation of the present study may be that there could also be anatomical and electrophysiological heterogeneity in apicobasal segments of the myocardium. However, the design of the study should have obviated this problem to some extent, as subjects with apical hypertrophy were excluded. The data presented do not permit a full assessment of the extent to which apicobasal versus anteroposterior gradients contribute to the ECG. Further studies are needed to address this point.

\section{Authors' affiliations}

K Sakata, M Shimizu, H Ino, M Yamaguchi, H Terai, K Hayashi, M Kiyama, T Hayashi, M Inove, H Mabuchi, Molecular Genetics of Cardiovascular Disorders, Division of Cardiovascular medicine, Graduate School of Medical Science, Kanazawa University, Kanazawa, Japan

\section{REFERENCES}

1 Cowan CJ, Yusoff K, Moore M, et al. Importance of lead selection in QT interval measurement. Am J Cardiol 1988:61:83-7.

2 Day CP, McComb JM, Campbell RWF. QT dispersion: an indication of arrhythmia risk in patients with long QT intervals. Br Heart J 1990;63:342-4.

3 Day CP, McComb JM, Campbell RWF. QT dispersion in sinus beats and ventricular extrasystoles in normal heart. Br Heart J 1992;67:39-4 1.

4 Dritsas A, Sbarouni E, Gilligan D, et al. QT-interval abnormalities in hypertrophic cardiomyopathy. Clin Cardiol 1992;15:739-42. 
5 Zaidi M, Robert A, Fesler R, et al. Dispersion of ventricular repolarization in hypertrophic cardiomyopathy. J Electrocardiol 1996;29(suppl):89-94.

6 Linker NJ, Colonna P, Kekwick CA, et al. Assessment of QT dispersion in symptomatic patients with congenital long QT syndromes. Am J Cardiol 1992:69:634-8

7 Perkiömäki JS, Koistinen M, Yli-Mäyry S, et al. Dispersion of the QT interval in patients with and without susceptibility to ventricular tachyarrhythmias after previous myocardial infarction. J Am Coll Cardiol 1995;26:174-9

8 Buja G, Miorelli M, Turrini P, et al. Comparison of QT dispersion in hypertrophic cardiomyopathy between patients with and without ventricular arrhythmias and sudden death. Am J Cardiol 1993:72:973-6

9 Miorelli $M$, Buja G, Melacini P, et al. QT-interval variability in hypertrophic cardiomyopathy patients with cardiac arrest. Int J Cardiol 1994;45: 121-7

10 Yetman AT, Hamilton RM, Benson LN, et al. Long-term outcome and prognostic determinants in children with hypertrophic cardiomyopathy. J Am Coll Cardiol 1998:32:1943-50.

11 Yi G, Elliot P, McKenna WJ, et al. QT dispersion and risk factors for sudden cardiac death in patients with hypertrophic cardiomyopathy. Am J Cardiol 1998;82:1514-9.
12 Maron BJ, Leyhe M, Casey SA et al. Assessment of QT dispersion as a prognostic marker for sudden death in a regional nonreferred hypertrophic cardiomyopathy cohort. Am J Cardiol 2001;87:114-15.

13 Maron BJ, Epstein SE. Hypertrophic cardiomyopathy. A discussion of nomenclature. Am J Cardiol 1979;43:1242-4.

14 Sahn DJ, de Maria A, Kisslo J, et al. Recommendations regarding quantitation in M-mode echocardiography: results of a survey of echocardiographic measurement. Circulation 1978;58:1072-83.

15 Mayet J, Shahi M, McGrath K, et al. Left ventricular hypertrophy and QT dispersion in hypertension. Hypertension 1996;28:791-6.

16 Ichkhan K, Molnar J, Somberg J. Relation of left ventricular mass and QT dispersion in patients with systematic hypertension. Am J Cardiol 1997:79:508-11.

17 Tomiyama $\mathbf{H}$, Doba N, Fu Y, et al. Left ventricular geometric patterns and QT dispersion in borderline and mild hypertension. Am J Hypertens 1998;11:286-92.

18 Spirito $\mathbf{P}$, Bellone $\mathrm{P}$, Harris KM, et al. Magnitude of left ventricular hypertrophy and risk of sudden death in hypertrophic cardiomyopathy. NEngl J Med 2000;342:1778-85.

19 Coumel P, Maison-Blanche P, Badilini F. Dispersion of ventricular repolarization. Reality? Illusion? Significance? Circulation 1998;97:2491-3.

\section{IMAGES IN CARDIOLOGY}

\section{latrogenic left coronary artery dissection}

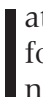

atrogenic left coronary dissection is fortunately rare. In this case haemodynamic collapse ensued necessitating the use of ionotropes and an intra-aortic balloon pump (top left panel). This stabilised the patient. There was no operating theatre free for urgent coronary bypass grafting. Introduction of a guidewire into the left anterior descending (LAD) coronary artery improved the situation, as did stenting of the left main stem (bottom left panel). The LAD and circumflex arteries were then stented in turn distal to proximal using eight stents in total (top right panel). The patient was well the following day with no troponin rise, and a normal 12 lead ECG and echocardiogram. Repeat angiography six months later showed only mild restenosis and the patient was pain-free with a normal exercise tolerance (bottom right panel).

W J Hobbs

A Chauhan

D Roberts

julian@hobbs 1430.fsnet.co.uk
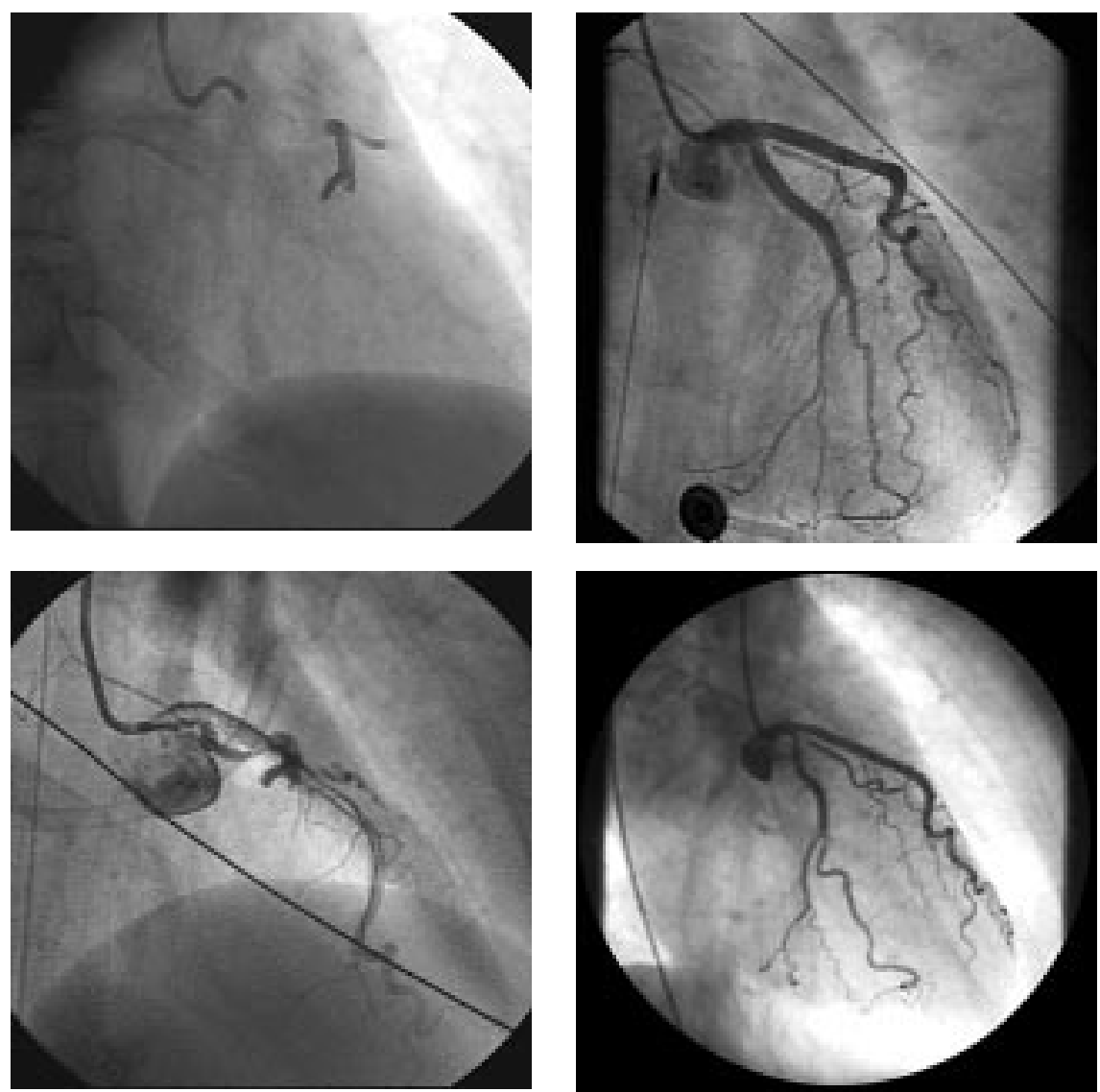